\title{
sciendo
}

Int. J. of Applied Mechanics and Engineering, 2019, vol.24, No.3, pp.661-673

DOI: 10.2478/ijame-2019-0041

\section{ON PROPAGATION OF RAYLEIGH TYPE SURFACE WAVE IN FIVE DIFFERENT THEORIES OF THERMOELASTICITY}

\author{
B. SINGH* \\ Department of Mathematics, Post Graduate Government College, Sector-11 \\ Chandigarh - 160011 , India \\ E-mail: bsinghgc11@gmail.com
}

S. VERMA

Hephzibah High School, 4558 Brothersville Road

Hephzibah, GA 30815, INDIA

E-mail: shailjav97@gmail.com

\begin{abstract}
The governing equations for a homogeneous and isotropic thermoelastic medium are formulated in the context of coupled thermoelasticity, Lord and Shulman theory of generalized thermoelasticity with one relaxation time, Green and Lindsay theory of generalized thermoelasticity with two relaxation times, Green and Nagdhi theory of thermoelasticity without energy dissipation and Chandrasekharaiah and Tzou theory of thermoelasticity. These governing equations are solved to obtain general surface wave solutions. The particular solutions in a halfspace are obtained with the help of appropriate radiation conditions. The two types of boundaries at athe surface of a half-space are considered namely, the stress free thermally insulated boundary and stress free isothermal boundary. The particular solutions obtained in a half-space satisfy the relevant boundary conditions at the free surface of the half-space and a frequency equation for the Rayleigh wave speed is obtained for both thermally insulated and isothermal cases. The non-dimensional Rayleigh wave speed is computed for aluminium metal to observe the effects of frequency, thermal relaxation time and different theories of thermoelasticity.
\end{abstract}

Key words: generalized thermoelasticity, surface waves, Rayleigh wave, frequency equation.

\section{Introduction}

The classical dynamical coupled theory of thermoelasticity with hyperbolic-parabolic field equations was developed by Biot [1]. This theory of thermoelasticity was extended by Lord and Shulman [2] and Green and Lindsay [3] and is termed as generalized thermoelasticity. Green and Naghdi [4] developed a theory of thermoelasticity without energy dissipation. These theories [2-4] use hyperbolic field equations for describing heat as a wave. The main difference between Biot's coupled thermoelasticity and generalized thermoelastic theories is that the generalized thermoelastic theories [2-4] admit a finite speed of heat propagation. Hetnarski and Ignaczak [5] and Ignaczak and Ostoja-Starzewski [6] reviewed these representative theories of generalized thermoelasticity.

Wave propagation phenomena have numerous applications in the fields of geophysical exploration, mineral and oil exploration and seismology. Plane wave propagation in thermoelasticity has many applications in various engineering fields. Problems on wave propagation in coupled or generalized thermoelasticity have been studied by various researchers [7-16]. The surface waves are very helpful for studying various aspects of an earthquake. In 1885, Lord Rayleigh [17] studied the propagation of surface waves along free surface of an isotropic elastic solid. Rayleigh waves are widely used for material characterization and to discover the mechanical and structural properties of the objects, because Rayleigh waves can travel along the surface of relatively thicker solid materials penetrating to a depth of one wave

\footnotetext{
* To whom correspondence should be addressed
} 
length and are very sensitive to surface defects. The studies on Rayleigh type surface wave in thermoelasticity are applicable in different engineering fields and future technologies. Various studies on Rayleigh surface wave in theory of thermoelasticity have been reported till date. Few of them are cited herein. For example, Lockett [18] studied the thermal effects on velocity of Rayleigh waves. Flavin [19] considered the propagation of thermo-elastic Rayleigh waves in a half space subjected to large uniform extensions at constant temperature in three mutually perpendicular directions. Chadwick and Windle [20] studied the effects of heat conduction upon the propagation of Rayleigh surface waves in a semi-infinite elastic solid in two special cases (i) when the surface of the solid is maintained at constant temperature and (ii) when the surface is thermally insulated. Tomita and Shindo [21] considered the propagation of Rayleigh waves in a perfectly conducting elastic half-space in the presence of magnetic fields. Dawn and Chakraborty [22] studied the Rayleigh wave in generalized thermoelastic media in the context of Green and Lindsay theory. Abd-Alla and Ahmed [23] investigated the influence both of gravity field and initial stress on the propagation of Rayleigh waves in an orthotropic thermoelastic medium. Ahmed [24] studied the effect of initial stress on the propagation of Rayleigh waves in a granular medium under incremental thermal stresses. Sharma et al. [25] presented an analysis of Rayleigh surface waves in a homogeneous, transversely isotropic, generalized piezothermoelastic half-space rotating with uniform angular velocity about normal to its boundary and subjected to stress free, electrically shorted/charge free and thermally insulated/isothermal boundary conditions. Abouelregal [26] studied the Rayleigh waves in a thermoelastic homogeneous isotropic solid half space in the context of dual-phase-lag model, where the medium is subjected to stress free, thermally insulated, boundary conditions. Mahmoud [27] investigated the influences of rotation, relaxation times, magnetic field, initial stress and gravity field on Rayleigh waves velocity in an elastic half-space of a granular medium. Chirita [28] studied the Rayleigh surface waves on an anisotropic homogeneous thermoelastic half-space. Singh [29] considered the propagation of Rayleigh waves in a thermoelastic solid half-space with microtemperatures. Bucur et al. [30] analyzed the behavior of plane harmonic waves and Rayleigh waves in a linear thermoelastic material with voids by considering the damped effects of the thermal field. Passarella et al. [31] considered the propagation of Rayleigh waves in isotropic strongly elliptic thermoelastic materials with microtemperatures in the context of Green and Naghdi theory. Biswas, et al. [32] studied the propagation of Rayleigh surface waves in a homogeneous, orthotropic thermoelastic half-space in the context of three-phase-lag model of thermoelasticity. Recently, Vashishth and Sukhija [33] investigated the propagation of coupled Rayleigh-type waves in a $2 \mathrm{~mm}$ piezoelectric layer over a porous piezo-thermoelastic half-space.

The aim of this paper is to study the propagation of Rayleigh type surface waves along the surface of an isotropic generalized thermoelastic solid half-space in the context of five different theories of thermoelasticity.

\section{Basic equations}

Following references [1-6], the linear governing equations of an isotropic and homogeneous thermoelastic solid in five different theories are:

the stress-strain-temperature relation

$$
\sigma_{i j}=\left[\lambda e-\gamma\left(1+v_{0} \frac{\partial}{\partial t}\right) T\right] \delta_{i j}+2 \mu e_{i j},
$$

the stress-displacement relation

$$
e_{i j}=\frac{1}{2}\left(\frac{\partial u_{i}}{\partial x_{j}}+\frac{\partial u_{j}}{\partial x_{i}}\right),
$$

the equation of motion 


$$
\rho \frac{\partial^{2} u_{i}}{\partial t^{2}}=\frac{\partial \sigma_{i j}}{\partial x_{j}}
$$

the heat conduction equation

$$
\kappa\left(n^{*}+t_{1} \frac{\partial}{\partial t}\right) \nabla^{2} T=\rho c_{e}\left(n^{*}+\tau_{0} \frac{\partial}{\partial t}\right) \frac{\partial T}{\partial t}+\gamma T_{0}\left(n_{1}+n_{0} \tau_{0} \frac{\partial}{\partial t}\right) \frac{\partial e}{\partial t} .
$$

Here, $\gamma=(3 \lambda+2 \mu) \alpha$ and $v_{0}$ and $\tau_{0}$ are the relaxation times which ensure a finite speed of heat propagation. Equations (2.3) and (2.4) reduce for the following five different theories as follows:

(a) Coupled thermoelastcity

If we put $n^{*}=n_{1}=1, t_{1}=\tau_{0}=v_{0}=0$, the field Eqs (2.3) and (2.4) are written as

$$
\begin{aligned}
& \sigma_{i j}=[\lambda e-\gamma T] \delta_{i j}+2 \mu e_{i j}, \\
& \kappa \nabla^{2} T=\rho c_{e} \frac{\partial T}{\partial t}+\gamma T_{0} \frac{\partial e}{\partial t} .
\end{aligned}
$$

(b) Lord-Shulman (L-S) theory

If we put $n^{*}=n_{1}=n_{0}=1, t_{1}=v_{0}=0, \tau_{0}>0$, the field Eqs (2.3) and (2.4) are written as

$$
\begin{aligned}
& \sigma_{i j}=[\lambda e-\gamma T] \delta_{i j}+2 \mu e_{i j}, \\
& \kappa \nabla^{2} T=\left(1+\tau_{0} \frac{\partial}{\partial t}\right)\left(\rho c_{e} \frac{\partial T}{\partial t}+\gamma T_{0} \frac{\partial e}{\partial t}\right) .
\end{aligned}
$$

(c) Green-Lindsay (G-L) theory

If we take $n^{*}=n_{1}=1, n_{0}=0, t_{1}=0, v_{0} \geq \tau_{0}>0$, the field Eqs (2.3) and (2.4) are written as

$$
\begin{aligned}
& \sigma_{i j}=\left[\lambda e-\gamma\left(1+v_{0} \frac{\partial}{\partial t}\right) T\right] \delta_{i j}+2 \mu e_{i j}, \\
& \kappa \nabla^{2} T=\rho c_{e}\left(1+\tau_{0} \frac{\partial}{\partial t}\right) \frac{\partial T}{\partial t}+\gamma T_{0} \frac{\partial e}{\partial t} .
\end{aligned}
$$

(d) Green-Naghdi type II (G-N-II) theory

If we take $n^{*}>0, n_{1}=0, n_{0}=1, t_{1}=v_{0}=0, \tau_{0}=1$, the field Eqs (2.3) and (2.4) are written as

$$
\begin{aligned}
& \sigma_{i j}=[\lambda e-\gamma T] \delta_{i j}+2 \mu e_{i j}, \\
& \kappa n^{*} \nabla^{2} T=\rho c_{e}\left(n^{*}+\frac{\partial}{\partial t}\right) \frac{\partial T}{\partial t}+\gamma T_{0} \frac{\partial^{2} e}{\partial t^{2}} .
\end{aligned}
$$

(e) Chandrasekharaiah and Tzou (C-T) theory [34] 
If we take $n^{*}=n_{1}=n_{0}=1, v_{0}=0, \tau_{0}>0, t_{1}>0$, the field Eqs (2.3) and (2.4) have the following form

$$
\begin{aligned}
& \sigma_{i j}=[\lambda e-\gamma T] \delta_{i j}+2 \mu e_{i j}, \\
& \kappa\left(1+t_{1} \frac{\partial}{\partial t}\right) \nabla^{2} T=\left(1+\tau_{0} \frac{\partial}{\partial t}\right)\left(\rho c_{e} \frac{\partial T}{\partial t}+\gamma T_{0} \frac{\partial e}{\partial t}\right) .
\end{aligned}
$$

\section{Method and solution}

For Rayleigh type waves in the half-space $z \geq 0$, the surface $z=0$ is assumed to be stress free. The present study is restricted to the plain strain parallel to the $x-z$ plane, with the displacement vector $u=\left(u_{1}, 0, u_{3}\right)$.

With the help of Eqs (2.1) and (2.2), Eqs (2.3) and (2.4) are written in the $x-z$ plane as

$$
\begin{aligned}
& \rho \frac{\partial^{2} u_{1}}{\partial t^{2}}=(\lambda+2 \mu) \frac{\partial^{2} u_{1}}{\partial x^{2}}+(\lambda+\mu) \frac{\partial^{2} u_{3}}{\partial x \partial z}+\mu \frac{\partial^{2} u_{1}}{\partial z^{2}}-\gamma\left(1+v_{0} \frac{\partial}{\partial t}\right) \frac{\partial T}{\partial x}, \\
& \rho \frac{\partial^{2} u_{3}}{\partial t^{2}}=(\lambda+2 \mu) \frac{\partial^{2} u_{3}}{\partial z^{2}}+(\lambda+\mu) \frac{\partial^{2} u_{1}}{\partial x \partial z}+\mu \frac{\partial^{2} u_{3}}{\partial x^{2}}-\gamma\left(1+v_{0} \frac{\partial}{\partial t}\right) \frac{\partial T}{\partial z}, \\
& \kappa\left(n^{*}+t_{1} \frac{\partial}{\partial t}\right)\left(\frac{\partial^{2} T}{\partial x^{2}}+\frac{\partial^{2} T}{\partial z^{2}}\right)=\rho c_{e}\left(n^{*}+\tau_{0} \frac{\partial}{\partial t}\right) \frac{\partial T}{\partial t}+\gamma T_{0}\left(n_{1}+n_{0} \tau_{0} \frac{\partial}{\partial t}\right)\left(\frac{\partial u_{1}}{\partial x \partial t}+\frac{\partial u_{3}}{\partial z \partial t}\right) .
\end{aligned}
$$

Using Helmohltz's representation, the displacement components $u_{1}$ and $u_{3}$ are written in terms of scalar potentials $\varphi$ and $\psi$ as

$$
u_{1}=\frac{\partial \varphi}{\partial x}-\frac{\partial \psi}{\partial z}, \quad u_{3}=\frac{\partial \varphi}{\partial z}+\frac{\partial \psi}{\partial x}
$$

Using Eq.(3.4) in Eqs (3.1) to (3.3), we obtain

$$
\begin{aligned}
& \rho \frac{\partial^{2} \varphi}{\partial t^{2}}=(\lambda+2 \mu)\left(\frac{\partial^{2} \varphi}{\partial x^{2}}+\frac{\partial^{2} \varphi}{\partial z^{2}}\right)-\gamma\left(1+v_{0} \frac{\partial}{\partial t}\right) T \\
& \rho \frac{\partial^{2} \psi}{\partial t^{2}}=\mu\left(\frac{\partial^{2} \psi}{\partial x^{2}}+\frac{\partial^{2} \psi}{\partial z^{2}}\right) \\
& \kappa\left(n^{*}+t_{1} \frac{\partial}{\partial t}\right)\left(\frac{\partial^{2} T}{\partial x^{2}}+\frac{\partial^{2} T}{\partial z^{2}}\right)=\rho c_{e}\left(n^{*}+\tau_{0} \frac{\partial}{\partial t}\right) \frac{\partial T}{\partial t}+\gamma T_{0}\left(n_{1}+n_{0} \tau_{0} \frac{\partial}{\partial t}\right) \frac{\partial}{\partial t}\left(\frac{\partial^{2} \varphi}{\partial x^{2}}+\frac{\partial^{2} \varphi}{\partial z^{2}}\right) .
\end{aligned}
$$

Here, Eqs (3.5) and (3.7) are coupled in $\varphi$ and $T$ and Eq.(3.6) is uncoupled. 
For thermoelastic waves in the half-space propagating in the $x$-direction, the functions $T, \varphi$ and $\psi$ are taken in the following form

$$
\{T, \varphi, \psi\}=\{\hat{T}(z), \hat{\varphi}(z), \hat{\psi}(z)\} \exp \imath(\eta x-\chi t)
$$

where $\chi^{2}=\eta^{2} c^{2}, \eta$ is the wave number and $\mathrm{c}$ is the phase velocity.

Substituting Eq.(3.8) in Eqs (3.5) to (3.7) and eliminating $\hat{T}, \hat{\varphi}$, we obtain the following auxiliary equation

$$
D^{4}-A D^{2}+B=0
$$

where

$$
\begin{aligned}
& D=d / d z \\
& A=-2 \eta^{2}-\eta^{2} c^{2}\left[-\frac{1}{c_{1}^{2}}+\frac{\left(\tau_{0}+n^{*} \frac{1}{\chi}\right)}{\bar{K} 1 \chi}-\frac{\varepsilon}{\bar{K}}\left(v_{0}+\frac{\imath}{\chi}\right)\left(n_{0} \tau_{0}+n_{1} \frac{\imath}{\chi}\right)\right], \\
& B=\eta^{4}+\eta^{4} c^{2}\left[-\frac{1}{c_{1}^{2}}+\frac{\left(\tau_{0}+n^{*} \frac{1}{\chi}\right)}{\bar{K} 1 \chi}-\frac{\varepsilon}{\bar{K}}\left(v_{0}+\frac{1}{\chi}\right)\left(n_{0} \tau_{0}+n_{1} \frac{1}{\chi}\right)\right]-\eta^{4} c^{4} \frac{\left(\tau_{0}+n^{*} \frac{1}{\chi}\right)}{\bar{K} 1 \chi c_{1}^{2}}, \\
& \varepsilon=\frac{\gamma^{2} T_{0}}{\rho^{2} c_{e} c_{1}^{2}}, \quad \bar{K}=\frac{\kappa}{\rho c_{e}}\left(t_{1}+n^{*} \frac{\mathrm{l}}{\chi}\right), \quad c^{2}=\frac{\chi^{2}}{\eta^{2}}, \quad c_{1}^{2}=\frac{(\lambda+2 \mu)}{\rho}, \quad c_{2}^{2}=\frac{\mu}{\rho} .
\end{aligned}
$$

With the help of Eq.(3.9) and keeping in mind that $\hat{\varphi}$ and $\hat{T} \rightarrow 0$ as $z \rightarrow \infty$ for surface waves, the solutions of $\varphi, T$ are written as

$$
\begin{aligned}
& \varphi(z)=\left[A \exp \left(-\eta \beta_{1} z\right)+B \exp \left(-\eta \beta_{2} z\right)\right] \exp \imath(\eta x-\chi t), \\
& T(z)=\frac{\rho c_{1}^{2}}{\gamma\left(1-\imath v_{0} \chi\right)} \exp \imath(\eta x-\chi t)\left[A\left\{\frac{\chi^{2}}{c_{1}^{2}}+\eta^{2}\left(\beta_{1}^{2}-1\right)\right\} \times\right. \\
& \left.\times \exp \left(-\eta \beta_{1} z\right)+B\left\{\frac{\chi^{2}}{c_{1}^{2}}+\eta^{2}\left(\beta_{2}^{2}-1\right)\right\} \times \exp \left(-\eta \beta_{2} z\right)\right]
\end{aligned}
$$

where

$$
\beta_{1}^{2}=\frac{1}{2 \eta^{2}}\left[A+\sqrt{A^{2}-4 B}\right], \quad \beta_{2}^{2}=\frac{1}{2 \eta^{2}}\left[A-\sqrt{A^{2}-4 B}\right]
$$

Using Eq.(3.8) in Eq.(3.6) and keeping in mind that $\hat{\psi} \rightarrow 0$ as $z \rightarrow \infty$ for surface waves, the solution of $\psi$ is written as 
where

$$
\psi(z)=C \exp \left(-\eta \beta_{3} z\right) \exp \imath(\eta x-\chi t)
$$

$$
\beta_{3}^{2}=1-\frac{c^{2}}{c_{2}^{2}}, \quad c_{2}^{2}=\frac{\mu}{\rho} .
$$

\section{Frequency equation}

\subsection{Isothermal case}

The mechanical and thermal boundary conditions at the stress free isothermal surface $z=0$ are

where

$$
\sigma_{z z}=0, \quad \sigma_{z x}=0, \quad T=0
$$

$$
\begin{aligned}
& \sigma_{z z}=(\lambda+2 \mu)\left\{\frac{\partial^{2} \varphi}{\partial z^{2}}+\frac{\partial^{2} \psi}{\partial x \partial z}\right\}+\lambda\left\{\frac{\partial^{2} \varphi}{\partial x^{2}}-\frac{\partial^{2} \psi}{\partial x \partial z}\right\}-\gamma\left(1+v_{0} \frac{\partial}{\partial t}\right) T \\
& \sigma_{z x}=\mu\left[\frac{\partial^{2} \psi}{\partial x^{2}}+2 \frac{\partial^{2} \varphi}{\partial x \partial z}-\frac{\partial^{2} \psi}{\partial z^{2}}\right] .
\end{aligned}
$$

Making use of solutions (3.10) to (3.12) and boundary conditions (4.1) and eliminating A, B and C, we obtain the following frequency equation

$$
\left(1+\beta_{3}^{2}\right)^{2}\left(\beta_{1}+\beta_{2}\right)+4 \beta_{3}\left\{\frac{c^{2}}{c_{1}^{2}}-\beta_{1} \beta_{2}-1\right\}=0 .
$$

\subsection{Thermally insulated case}

The mechanical and thermal boundary conditions at the thermally insulated surface $z=0$ are

$$
\sigma_{z z}=0, \quad \sigma_{z x}=0, \quad \frac{\partial T}{\partial z}=0 .
$$

Making use of solutions (3.10) to (3.12) and boundary conditions (4.3) and eliminating A, B and C, we obtain the following frequency equation

$$
\begin{aligned}
& (\lambda+2 \mu)\left(1+\beta_{3}^{2}\right) \beta_{1} \beta_{2}\left(\frac{c^{2}}{c_{1}^{2}}+\beta_{1} \beta_{2}-1\right)+\lambda\left(1+\beta_{3}^{2}\right)\left\{\frac{c^{2}}{c_{1}^{2}}+\beta_{1}^{2}+\beta_{2}^{2}+\beta_{1} \beta_{2}-1\right\}+ \\
& +\rho c_{1}^{2}\left(1+\beta_{3}^{2}\right)\left(\frac{c^{2}}{c_{1}^{2}}+\beta_{1}^{2}-1\right)\left(\frac{c^{2}}{c_{1}^{2}}+\beta_{2}^{2}-1\right)+4 \mu \beta_{1} \beta_{2} \beta_{3}\left(\beta_{1}+\beta_{2}\right)=0 .
\end{aligned}
$$

\section{Special cases}

\subsection{Small thermal coupling $(\varepsilon<<1)$}

For $(\varepsilon<<1)$ the approximated expressions for $\beta_{1}$ and $\beta_{2}$ are obtained as 


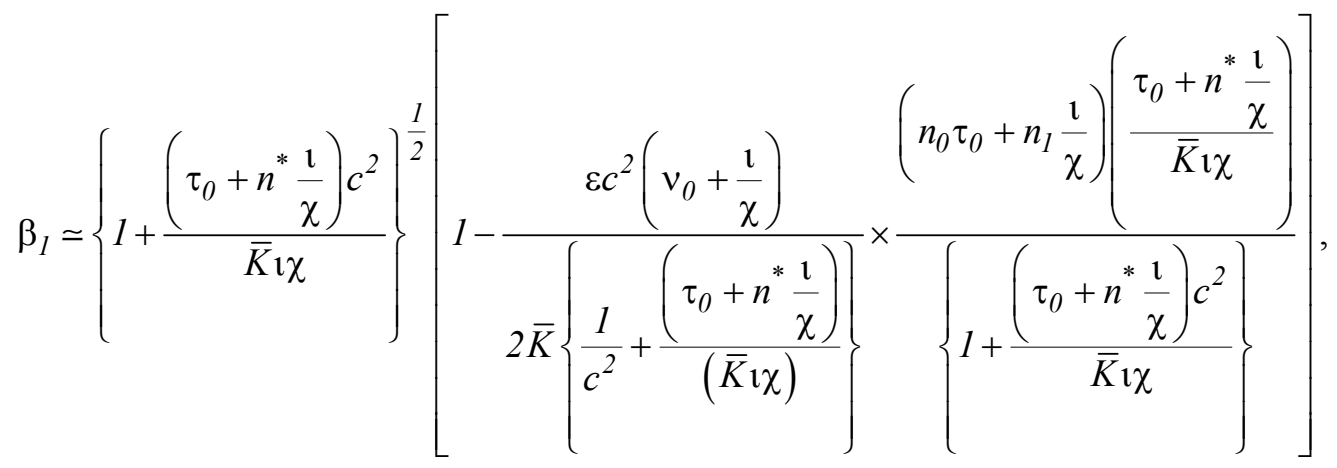

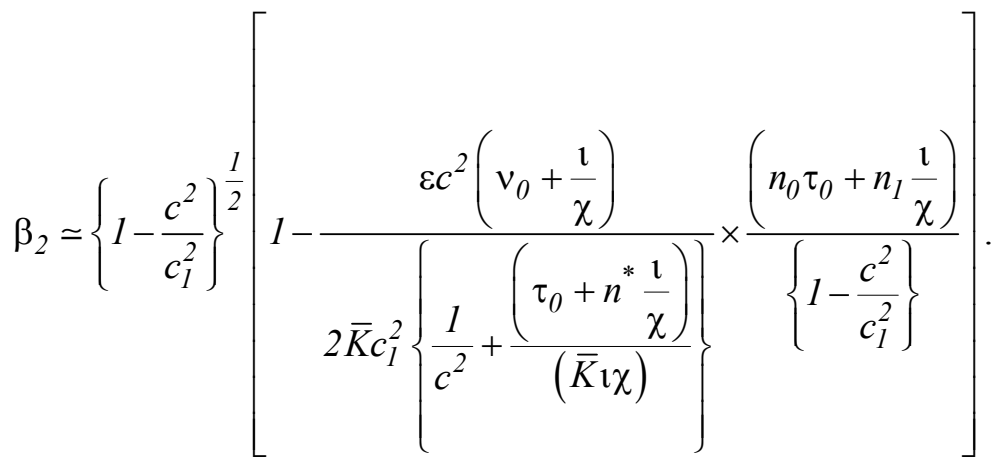

\subsection{Small reduced frequency $(\chi<<1)$}

For small reduced frequency $\chi<<1$, the approximated expressions for $\beta_{1}$ and $\beta_{2}$ are obtained as

$$
\begin{aligned}
& \beta_{1} \simeq \frac{1}{\sqrt{\chi}} c\left(\frac{\tau_{0}+n^{*} \frac{\mathrm{\imath}}{\chi}}{\bar{K} \mathrm{l}}\right)^{\frac{1}{2}}\left[1+\frac{\chi}{2 c^{2}\left(\frac{\tau_{0}+n^{*} \frac{\mathrm{\imath}}{\chi}}{\bar{K} \mathrm{l}}\right)}\left\{1-\frac{\varepsilon c^{2}}{\bar{K}}\left(v_{0}+\frac{\mathrm{\imath}}{\chi}\right)\left(n_{0} \tau_{0}+n_{1} \frac{\mathrm{l}}{\chi}\right)\right\}\right] \\
& \beta_{2}=\left(1-\frac{c^{2}}{c_{1}^{2}}\right)^{\frac{1}{2}} .
\end{aligned}
$$

\subsection{Isotropic elastic case}

If we neglect thermal parameters, then the frequency Eqs (4.2) and (4.4) reduce to

$$
\left(2-\frac{c^{2}}{c_{1}^{2}}\right)^{2}=4 \sqrt{1-\frac{c^{2}}{c_{1}^{2}}} \sqrt{1-\frac{c^{2}}{c_{2}^{2}}},
$$

which is the velocity equation of thr Rayleigh wave along the surface of an isotropic elastic half-space. 


\section{Numerical results and discussion}

The following physical constants of aluminium metal are chosen to compute the non-dimensional speed $c / c_{2}$ of the Rayleigh wave in a thermoelastic solid half-space in the context of five different theories of thermoelastcity

$$
\begin{aligned}
& \rho=2.7 \mathrm{gm} . \mathrm{cm}^{-3}, \quad \lambda=5.8 \times 10^{11} \text { dyne.cm }{ }^{-2}, \quad \mu=2.6 \times 10^{11} \text { dyne.cm }^{-2}, \\
& K=0.5 \mathrm{cal} . \mathrm{cm}^{-1} \cdot \mathrm{s}^{-1} \cdot{ }^{o} \mathrm{C}^{-1}, \quad c_{e}=0.9 \mathrm{cal} . \mathrm{gm}^{-1} \cdot{ }^{o} \mathrm{C}^{-1}, \mathrm{v}_{0}=0.0006 \mathrm{~s}, \quad t_{1}=0.0006 \mathrm{~s}
\end{aligned}
$$

The frequency Eqs (4.2) and (4.4) are solved numerically for the real part of non-dimensional speed $c / c_{2}$ of Rayleigh waves by using a program of an iteration method. The variations of non-dimensional speed $\left(c / c_{2}\right)$ of Rayleigh wave against the frequency $(\chi)$ are shown by a solid line (coupled thermoelasticity), solid line with rhombus (L-S theory), solid line with triangle (G-L theory), solid line with stars (G-N-II theory) and solid line with circles (C-T theory) in Figs 1 and 2 for isothermal and thermally insulated cases, respectively, when $\tau_{0}=0.0005 \mathrm{~s}$.

For coupled thermoelasticity in Fig.1 (isothermal case), the non-dimensional wave speed is 0.92934263 at $\chi=1 \mathrm{~Hz}$. The wave speed increases first sharply with the increase in value of frequency and then slowly to a value 0.9416163 at $\chi=20 \mathrm{~Hz}$. The variations in cases of the Lord and Shulman (L-S) theory, Green and Lindsay (G-L) theory and Chandrasekharaiah and Tzou (C-T) theory are similar to that of coupled thermoelasticity. The values of non-dimensional wave speed of the Rayleigh wave in these theories are different in 3rd and 4th decimal places at each value of frequency. In the case of the Green and Naghdi (G-N-II) theory, the non-dimensional wave speed is 0.926241 at each value of frequency.

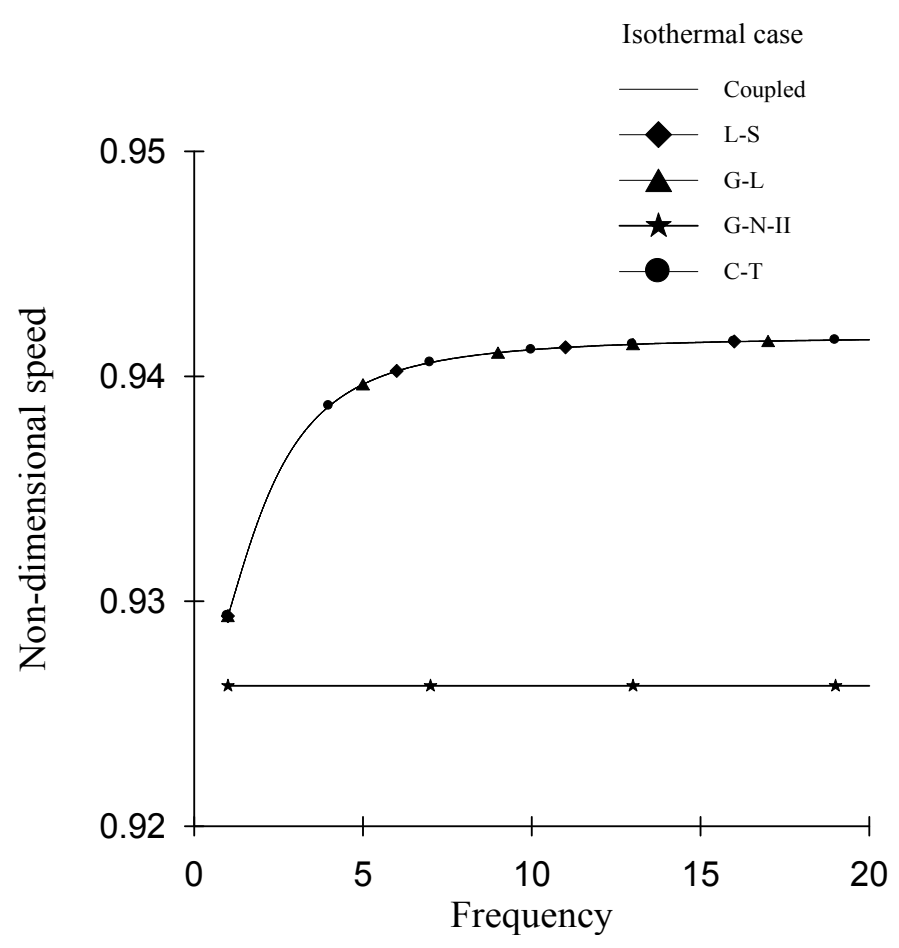

Fig.1. Variations of non-dimensional speed $\left(c / c_{2}\right)$ of Rayleigh wave against the frequency $(\chi)$ in five different theories of thermoelasticity for isothermal case. 
For coupled thermoelasticity in Fig.2 (thermally insulated case), the non-dimensional wave speed is 0.85341489 at $\chi=1 \mathrm{~Hz}$. The wave speed increases with the increase in value of frequency and attains a value 1.01074839 at $\chi=20 \mathrm{~Hz}$. The variations in cases of the Lord and Shulman (L-S) theory, Green and Lindsay (G-L) theory and Chandrasekharaiah and Tzou (C-T) theory are also similar to that of coupled thermoelasticity. However, the values of non-dimensional wave speed of the Rayleigh wave in these theories are same up to 2nd or 3rd decimal places at each value of frequency. In the case of Green and Naghdi (G-NII) theory, the non-dimensional wave speed is 0.8510 at each value of frequency.

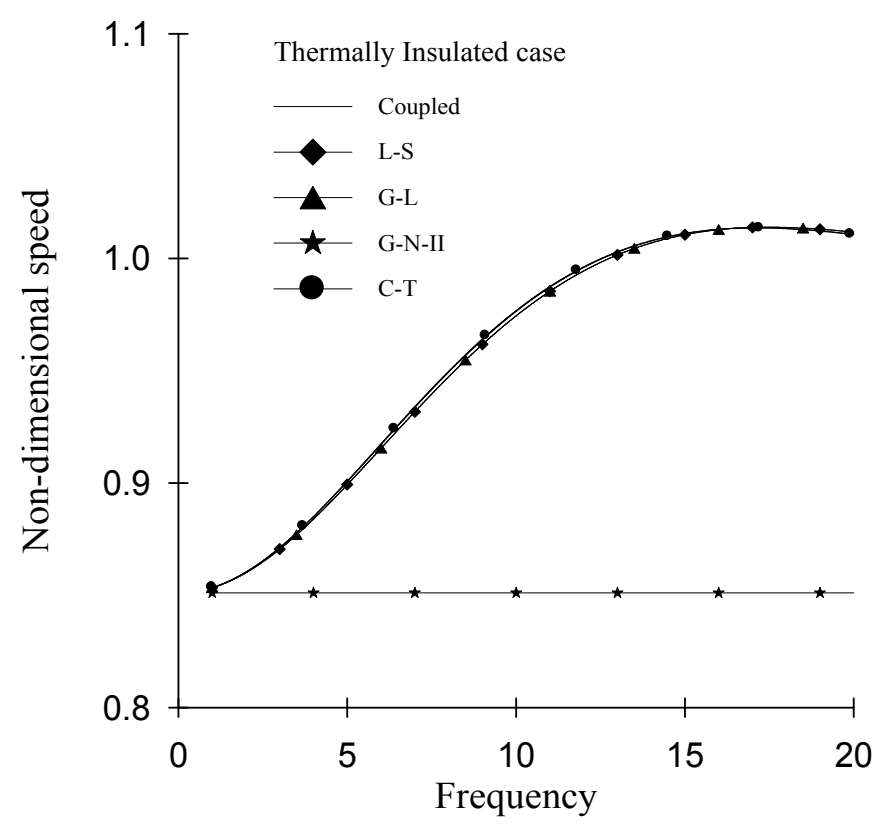

Fig.2. Variations of non-dimensional speed $\left(c / c_{2}\right)$ of Rayleigh wave against the frequency $(\chi)$ in five different theories of thermoelasticity for thermally insulated case.

In Green-Naghdi theory of type-II, the variations of non-dimensional speed $\left(c / c_{2}\right)$ of the Rayleigh wave against the parameter $n^{*}$ are shown in Fig.3 by solid and dashed lines for isothermal and thermally insulated cases, respectively, when $\chi=10 \mathrm{~Hz}$ and $\tau_{0}=0.0005$. For the isothermal case, the non-dimensional value of wave speed is 0.92624146 at $n^{*}=0.001$. It decreases slowly to a value of 0.91144258 at $n^{*}=1$. For the thermally insulated case, the non-dimensional value of wave speed is 0.85121471 at $n^{*}=0.001$. It increases sharply to a maximum value 0.99999964 at $n^{*}=0.868$ and then decreases to a value of 0.98975891 at $n^{*}=1$. 


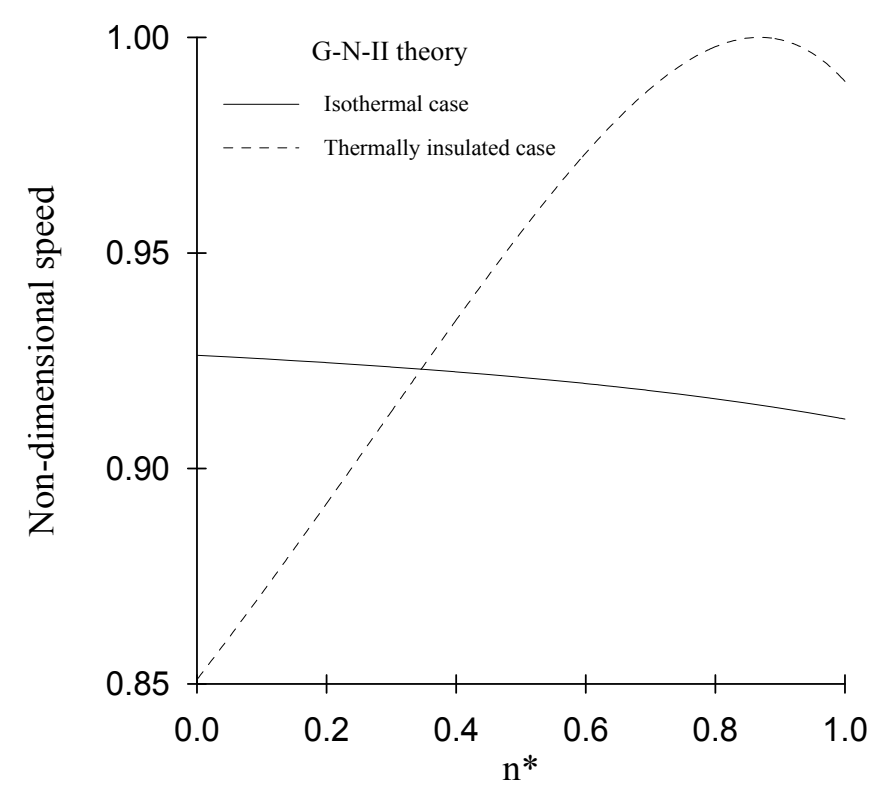

Fig.3. Variations of non-dimensional speed $\left(c / c_{2}\right)$ of Rayleigh wave against a parameter $\left(n^{*}\right)$ in GreenNaghdi theory of type-II for both thermally insulated and isothermal cases.

In C-T theory of thermoelasticity, the variations of non-dimensional speed $\left(c / c_{2}\right)$ of the Rayleigh wave against the thermal relaxation time $\left(\tau_{0}\right)$ are shown in Fig.4 by solid and dashed lines for isothermal and thermally insulated cases, respectively, when $\chi=10 \mathrm{~Hz}$. For the isothermal case, the value of non-dimensional wave speed is 0.94118643 at $\tau_{0}=0.001 \mathrm{~s}$. It increases slowly and attains a value of 0.94447386 at $\tau_{0}=0.1 \mathrm{~s}$. For the thermally insulated case, the non-dimensional value of wave speed is 0.96714586 at $\tau_{0}=0.001$. It increases to a maximum value 1.0030185 at $\tau_{0}=0.071 \mathrm{~s}$ and then decreases to a value of 1.00000036 at $\tau_{0}=0.1 \mathrm{~s}$.

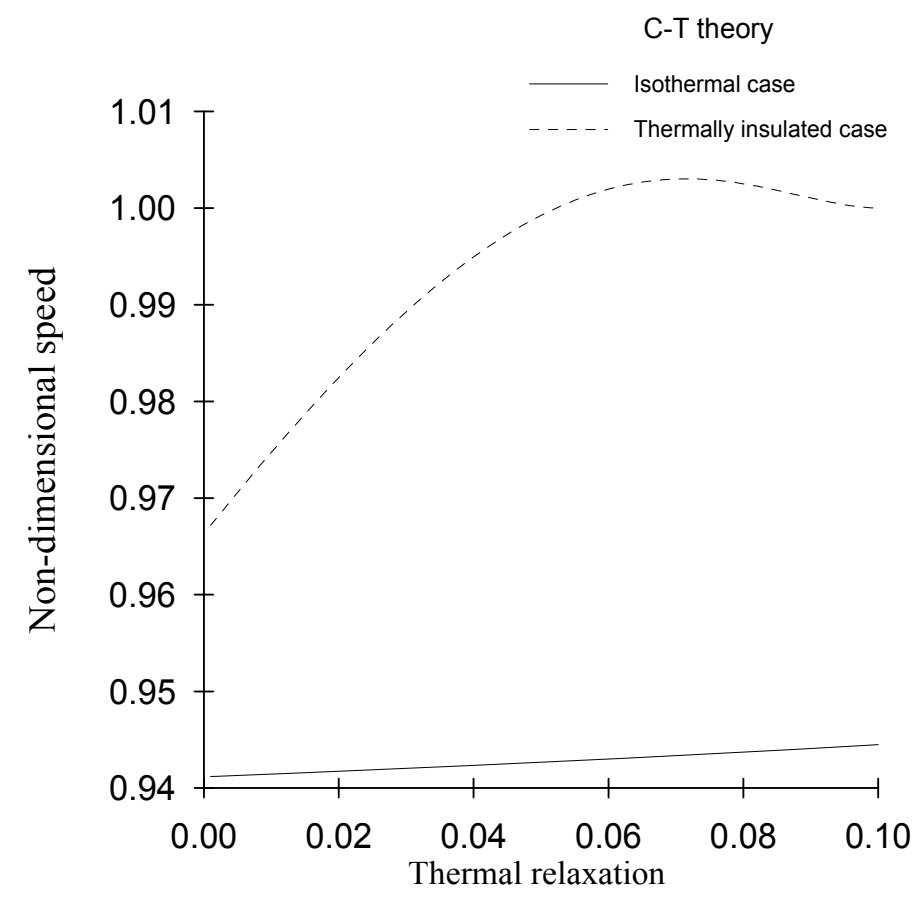

Fig.4. Variations of non-dimensional speed $\left(c / c_{2}\right)$ of Rayleigh wave against the thermal relaxation time $\tau_{0}$ in C-T theory for both thermally insulated and isothermal cases. 


\section{Conclusions}

A problem on propagation of Rayleigh surface wave is solved in the context of coupled thermoelasticity, Lord and Shulman theory of generalized thermoelasticity with one relaxation time, Green and Lindsay theory of generalized thermoelasticity with two relaxation times, Green and Nagdhi theory of thermoelasticity without energy dissipation and Chandrasekharaiah and Tzou theory of thermoelasticity. Using appropriate radiation conditions, the surface wave solutions in a thermoelasic solid half-space are obtained. A frequency equation of the Rayleigh surface wave is obtained for both thermally insulated and isothermal boundaries. Using the frequency Eqs (4.2) and (4.4), the non-dimensional wave speed of the Rayleigh surface wave is computed for mechanical and thermal constants of aluminium metal. The nondimensional speed of the Rayleigh wave is plotted against frequency, parameter $n^{*}$ and thermal relaxation time $\tau_{0}$. The numerical results are discussed in detail. The following important observations are made from the numerical results:

(i) For the isothermal case in Fig.1 and for the thermally insulated case in Fig.2, the effect of frequency on the non-dimensional wave speed is observed significant in coupled thermoelasticity, L-S, G-L and C-T theories, whereas the effect of frequency on the non-dimensional wave speed is negligible in the case of G-N-II theory.

(ii) The comparison of solid and dashed variations in Fig.3 shows the effect of G-N theory parameter $n^{*}$ and thermal boundary on the non-dimensional wave speed of the Rayleigh wave.

(iii) The comparison of solid and dashed variations in Fig. 4 shows the effect of the thermal relaxation time $\tau_{0}$ and thermal boundary on the non-dimensional wave speed of the Rayleigh wave in C-T theroy.

\section{Acknowledgement}

One of the Authors, Baljeet Singh is grateful to University Grants Commission, New Delhi for granting a Major Research Project (MRP-MAJOR-MATH-2013-2149).

\section{Nomenclature}

$$
\begin{aligned}
c & - \text { phase velocity } \\
c_{e} & - \text { specific heat at constant strain } \\
e=u_{i, i} & - \text { dilatation } \\
e_{i j} & - \text { Cartesian components of the linear strain tensor } \\
n^{*} & - \text { parameter in Green-Nagdhi theory } \\
n_{1}, n_{0}, t_{1} & - \text { parameters } \\
T & - \text { the change in the absolute basic temperature } T_{0} \\
t & - \text { time } \\
u_{i} & - \text { displacement components } \\
x_{i} & - \text { Cartesian coordinates } \\
\alpha & - \text { coefficient of thermal expansion } \\
\delta_{i j} & - \text { Kronecker delta } \\
\eta & - \text { wave number } \\
\kappa & - \text { coefficient of thermal conductivity } \\
\lambda, \mu & - \text { the Lame's constants } \\
v_{0}, \tau_{0} & - \text { the relaxation times } \\
\rho & - \text { coefficient of mass density }
\end{aligned}
$$


$\tau_{i j} \quad$ - Cartesian components of the linear stress tensor

$\varphi, \psi-$ scalar potentials

$\nabla^{2}$ - Laplace operator

\section{References}

[1] Biot M.A. (1956): Thermoelasticity and irreversible thermodynamics. - J. Appl. Phys., vol.2, pp.240-253.

[2] Green A.E. and Lindsay K.A. (1972): Thermoelasticity. - J. Elasticity, vol.2, pp.1-7.

[3] Lord H. and Shulman Y. (1967): A generalised dynamical theory of thermoelasticity. - J. Mech. Phys. Solids, vol.15, pp.299-309.

[4] Green A.E. and Naghdi P.M. (1993): Thermoelasticity without energy dissipation. - J. Elast., vol.31, pp.189-208.

[5] Hetnarski R.B. and Ignaczak J. (1999): Generalized thermoelasticity. - J. Thermal Stresses, vol.22, pp.451-476.

[6] Ignaczak J. and Ostoja-Starzewski M. (2009): Thermoelasticity with Finite Wave Speeds. - Oxford University Press.

[7] Deresiewicz H. (1960): Effect of boundaries on waves in a thermo-elastic solid: Reflection of plane waves from plane boundary. - J. Mech. Phys. Solids, vol.8, pp.164-172.

[8] Sinha A.N. and Sinha S.B. (1974): Reflection of thermoelastic waves at a solid half space with thermal relaxation. - J. Phys. Earth, vol.22, pp.237-244.

[9] Sinha S.B. and Elsibai K.A. (1996): Reflection of thermoelastic waves at a solid half-space with two thermal relaxation times. - J. Thermal Stresses, vol.19, pp.763-777.

[10] Sinha S.B. and Elsibai K.A. (1997): Reflection and refraction of thermoelastic waves at an interface of two semiinfinite media with two thermal relaxation times. - J. Thermal Stresses, vol.20, pp.129-146.

[11] Sharma J.N., Kumar V. and Chand D. (2003): Reflection of generalized thermoelastic waves from the boundary of a half-space. - J. Thermal Stresses, vol.26, pp.925-942.

[12] Singh B. (2008): Effect of hydrostatic initial stresses on waves in a thermoelastic solid half-space. - Applied Math. Comp., vol.198, pp.494-505.

[13] Singh B. (2010): Reflection of plane waves at the free surface of a monoclinic thermoelastic solid half-space. European J. Mech. A-Solids, vol.29, pp.911-916.

[14] Singh M.C. and Chakraborty N. (2015): Reflection of a plane magneto-thermoelastic wave at the boundary of a solid half-space in presence of initial stress. - Appl. Math. Modelling, vol.39, pp.1409-1421.

[15] Wei W., Zheng R., Liu G. and Tao H (2016): Reflection and refraction of $P$ wave at the interface between thermoelastic and porous thermoelastic medium. - Transport in Porous Media, vol.113, pp.1-27.

[16] Li Y., Li L., Wei P. and Wang C. (2018): Reflection and refraction of thermoelastic waves at an interface of two couple-stress solids based on Lord-Shulman thermoelastic theory. - Appl. Math. Modelling, vol.55, pp.536-550.

[17] Rayleigh L. (1885): On waves propagated along the plane surface of an elastic solid. - Proc. R. Soc. London, Ser. A, vol.17, pp.4-11.

[18] Lockett F.J. (1958): Effect of the thermal properties of a solid on the velocity of Rayleigh waves. - J. Mech. Phys. Solids, vol.7, pp.71-75.

[19] Flavin J.N. (1962): Thermoelastic Rayleigh waves in a prestressed medium. - Math. Proc. Cambridge Phil. Soc., vol.58, pp.532-538. 
[20] Chadwick P. and Windle D.W. (1964): Propagation of Rayleigh waves along isothermal and insulated boundaries. - Proc. R. Soc. Lond. A, vol.280, pp.47-71.

[21] Tomita S. and Shindo Y. (1979): Rayleigh waves in magneto-thermoelastic solids with thermal relaxation. - Int. J. Eng. Sci, vol.17, pp.227-232.

[22] Dawn N.C. and Chakraborty S.K. (1988): On Rayleigh wave in Green-Lindsay's model of generalized thermoelastic media. - Ind. J. Pure Appl. Math, vol.20, pp.273-286.

[23] Abd-Alla A.M. and Ahmed M. (1996): Rayleigh waves in an orthotropic thermoelastic medium under gravity field and initial stress. - Earth, Moon, and Planets, vol.75, pp.185-197.

[24] Ahmed S.M. (2000): Rayleigh waves in a thermoelastic granular medium under initial stress. - Int. J. Math. Math. Sci., vol.23, pp.627-637.

[25] Sharma J.N., Walia V. and Gupta S.K. (2008): Effect of rotation and thermal relaxation on Rayleigh waves in piezothermoelastic half space. - Int. J. Mech. Sci., vol.50, pp.433-444.

[26] Abouelregal A. E. (2011): Rayleigh waves in a thermoelastic solid half space using dual-phase-lag model. - Int. J. Eng. Sci., vol.49, pp.781-791.

[27] Mahmoud S.R. (2012): Influence of rotation and generalized magneto-thermoelastic on Rayleigh waves in a granular medium under effect of initial stress and gravity field. - Meccanica, vol.47, pp.1561-1579.

[28] Chirita S. (2013): On the Rayleigh surface waves on an anisotropic homogeneous thermoelastic half-space. Acta Mech., vol.224, pp.657-674.

[29] Singh B. (2014): Propagation of Rayleigh wave in a thermoelastic solid half-space with microtemperatures. Int. J. Geophys., Article ID 474502, 6 pages, http://dx.doi.org/10.1155/2014/474502 (2014),

[30] Bucur A.V., Passarella F. and Tibullo V. (2014): Rayleigh surface waves in the theory of thermoelastic materials with voids. - Meccanica, vol.49, pp.2069-2078.

[31] Passarella F., Tibullo V. and Viccione G. (2017): Rayleigh waves in isotropic strongly elliptic thermoelastic materials with microtemperatures. - Meccanica, vol.52, pp.3033-3041.

[32] Biswas S., Mukhopadhyay B. and Shaw S. (2017): Rayleigh surface wave propagation in orthotropic thermoelastic solids under three-phase-lag model. - J. Thermal Stresses, vol.40, pp.403-419.

[33] Vashishth A.K. and Sukhija H. (2017): Coupled Rayleigh waves in a 2-mm piezoelectric layer over a porous piezo-thermoelastic half-space. - Acta Mechanica, vol.228, pp.773-803.

[34] Tzou D.Y. (1995): A unified approach for heat conduction from macro to micro-scales. - J. Heat Transfer, vol.117, pp.8-16.

Received: January 26, 2018

Revised: June 6, 2018 